\title{
Cryptosporidium and Giardia taxa in faecal samples from animals in catchments supplying the city of Melbourne with drinking water (2011 to 2015)
}

\author{
Anson V. Koehler ${ }^{1 *}$, Shane R. Haydon ${ }^{2}$, Aaron R. Jex ${ }^{1,3}$ and Robin B. Gasser ${ }^{1 *}$
}

\begin{abstract}
Background: In a long-term program to monitor pathogens in water catchments serving the City of Melbourne in the State of Victoria in Australia, we detected and genetically characterised Cryptosporidium and Giardia in faecal samples from various animals in nine water reservoir areas over a period of 4 years (July 2011 to November 2015).

Methods: This work was conducted using PCR-based single-strand conformation polymorphism (SSCP) and phylogenetic analyses of portions of the small subunit of ribosomal RNA (SSU) and $60 \mathrm{kDa}$ glycoprotein (gp60) genes for Cryptosporidium, and triose-phosphate isomerase (tpi) gene for Giardia.

Results: The prevalence of Cryptosporidium was $1.62 \%$ (69 of 4,256 samples); 25 distinct sequence types were defined for pSSU, and six for gp60 which represented C. hominis (genotype lb - subgenotype IbA10G2), C. cuniculus (genotype Vb - subgenotypes VbA26, and VbA25), and C. canis, C. fayeri, C. macropodum, C. parvum, C. ryanae, Cryptosporidium sp. "duck" genotype, C. suis and C. ubiquitum as well as 12 novel SSU sequence types. The prevalence of Giardia was $0.31 \%$ (13 of 4,256 samples); all three distinct tpi sequence types defined represented assemblage A of G. duodenalis.

Conclusions: Of the 34 sequence types (genotypes) characterized here, five and one have been recorded previously for Cryptosporidium and Giardia, respectively, from humans. Novel genotypes of Cryptosporidium and Giardia were recorded for SSU $(n=12)$, gp60 $(n=4)$ and tpi $(n=1)$; the zoonotic potential of these novel genotypes is presently unknown. Future work will continue to monitor the prevalence of Cryptosporidium and Giardia genotypes in animals in these catchments, and expand investigations to humans. Nucleotide sequences reported in this paper are available in the GenBank database under accession nos. KU531647-KU531718.
\end{abstract}

Keywords: Cryptosporidium, Giardia, 60 kDa glycoprotein (gp60) gene, Small subunit (SSU) of ribosomal RNA gene, Single-strand conformation polymorphism (SSCP), Triose-phosphate isomerase (tpi) gene

\section{Background}

One of the toughest challenges facing the world's supply of clean drinking water is contamination from faeces and soil [1,2]. Diarrhoeal disease is responsible for $10.5 \%$ of deaths in children of less than five years of age [3-5], having a greater impact than malaria and HIV/ AIDS combined [5]. Pathogens of concern include viruses, bacteria and protists [1]. Of the latter pathogen

\footnotetext{
* Correspondence: anson.koehler@unimelb.edu.au; robinbg@unimelb.edu.au ${ }^{1}$ Faculty of Veterinary and Agricultural Sciences, The University of Melbourne, Parkville, Victoria 3010, Australia

Full list of author information is available at the end of the article
}

group, human-infective taxa (i.e. species and genotypes/assemblages) of Cryptosporidium and Giardia are highly significant $[1,4-8]$. Cryptosporidium and Giardia are unique in that very small numbers of infective stages (oocysts and cysts, respectively) can cause disease in humans $[9,10]$ and that these stages are resistant to chlorination and other common water treatments $[1,11]$. An example of the tremendous impact these parasites can have was demonstrated in 1993, with a major outbreak of cryptosporidiosis in Milwaukee, USA [12], which affected more than 400,000 people and resulted in 100 deaths. This case emphasizes the major public health 
importance of waterborne diseases and the need for their sustained prevention.

Melbourne (Victoria, Australia; population 4 million) is one of the few cities in the world that receives largely unfiltered drinking water from protected wilderness catchment areas. The management of Melbourne's ten main water catchment areas includes restricted access for humans, long water retention times and an intense program of testing and monitoring for pathogens in source water. These catchments represent habitat for native and feral animals, such that the monitoring of zoonotic pathogens is central to management and the prevention of outbreaks of waterborne disease. In 2008, we initiated a program to monitor Cryptosporidium and Giardia in faecal samples from various mammals and birds in the Melbourne's catchments [13]. To do this, we collected 2,009 fresh faecal samples (from June 2009 to June 2011) and tested them using an established and validated PCR-based mutation scanningcoupled sequencing approach (cf. [14-16]), combined with phylogenetic analyses of loci (SSU and gp60) in the small subunit $(S S U)$ of ribosomal RNA and $60-\mathrm{kDa}$ glycoprotein (gp60) genes to detect and characterise Cryptosporidium, and another locus (tpi) in the triose-phosphate isomerase (tpi) gene to identify and classify Giardia [13]. In total, Cryptosporidium and Giardia were detected in 2.8 and $3.4 \%$ of all 2009 samples tested, respectively, and 35 previously undescribed genotypes were reported [13]. In spite of this relatively low prevalence, the findings from this study emphasized a need for a sustained program.

Therefore, from July 2011 to November 2015, we extended our monitoring program, and genetically characterised Cryptosporidium and Giardia from native and introduced animals in Melbourne's water catchments, in order to continually assess the prevalence and diversity of Cryptosporidium and Giardia taxa, evaluate their host affiliations, geographical distributions and zoonotic potential, and support catchment management. In the present article, we describe the results from this 4-year study and discuss the findings in a water industry context.

\section{Methods}

\section{Melbourne's catchments}

Greater Melbourne sources its municipal drinking water from ten main water catchment reservoirs. Approximately $80 \%$ of Melbourne's drinking water is drawn from 'closed' catchments in the Yarra Ranges $(\sim 85 \mathrm{~km}$ east of Melbourne), which cover 157,000 hectares of eucalypt forest, with restricted human and domestic animal access, to minimise the risk of waterborne diseases. The remaining $20 \%$ of Melbourne's water comes from 'open' catchments, in which some farming and human activities are permitted. All water undergoes treatment in accordance with national and international guidelines $[17,18]$. The nine reservoirs studied here (Fig. 1) are located north and east of Melbourne's central business district (CBD), are less than $90 \mathrm{~km}$ apart and include: Cardinia (CA) $37^{\circ} 47^{\prime} \mathrm{S}, 145^{\circ} 24^{\prime} \mathrm{E}$; Greenvale (GV) $37^{\circ} 37^{\prime} \mathrm{S}$, $144^{\circ} 54^{\prime} \mathrm{E}$; Maroondah (MR) $37^{\circ} 38^{\prime} \mathrm{S}, 145^{\circ} 33^{\prime} \mathrm{E}$; O’Shannassy (OS) $37^{\circ} 40^{\prime} \mathrm{S}, 1^{\circ} 45^{\prime} \mathrm{E}$; Silvan (SV) $37^{\circ} 50^{\prime} \mathrm{S}, 1^{\circ} 5^{\circ} 25^{\prime} \mathrm{E}$; Tarago (TAR) $37^{\circ} 59^{\prime} \mathrm{S}, 145^{\circ} 55^{\prime} \mathrm{E}$; Thompson (TH) $37^{\circ} 47^{\prime} \mathrm{S}$, $146^{\circ} 21^{\prime} \mathrm{E}$; Upper Yarra (UY) $37^{\circ} 40^{\prime} \mathrm{S}, 1^{\circ} 5^{\circ} 55^{\prime} \mathrm{E}$; and Yan Yean (YY) $37^{\circ} 33^{\prime} S, 145^{\circ} 08^{\prime} E$. Reservoirs MR, OS, TH and UY are situated in the densely forested Yarra Ranges catchment, whereas YY reservoir is a much smaller catchment north of the CBD and surrounded by residential and grazing land. The remaining reservoirs, including CA, GV and SV, act as storage facilities for the larger catchments and have eucalypt and/or pine forests. TAR is the one 'open' water supply catchment, which permits farming in the land surrounding the reservoir. All regions have small areas of grassland adjacent to water reservoirs, and it is here that faecal samples were collected.

\section{Samples and isolation of genomic DNA}

A total of 4,256 faecal deposits from Canis familiaris (dog), Vulpes vulpes (fox), Felis catus (cat), Dromaius novaehollandiae (emu), Wallabia bicolor (swamp wallaby), Macropus giganteus (Eastern grey kangaroo), Trichosurus vulpecula (common brushtail possum), Oryctolagus cuniculus (rabbit), Rattus lutreolus (swamp rat), Rattus fuscipes (bush rat) and Vombatus ursinus (common wombat), together with waterbirds, principally the Australian wood duck (Chenonetta jubata), and deer, including Rusa unicolour (sambar), Cervus elaphus (red) and Dama dama (fallow), and samples of unknown host origin were collected from nine locations from July 2011 to November 2015 (see Additional file 1: Table S1). Specifically, samples were collected from CA $(n=718), \mathrm{GV}(n=638), \mathrm{MR}$ $(n=344)$, OS $(n=622)$ SV $(n=527)$, TAR $(n=61)$, TH $(n=31)$, UY $(n=714)$ and YY $(n=601)$. Scats were identified using a field guide [19], and host identity was confirmed, as required, by PCR-based sequencing of a region of the mitochondrial cytochrome $b$ gene from faecal DNA using a similar approach to that described by Dalen et al. [20]. Genomic DNA was extracted directly from $0.25 \mathrm{~g}$ of faeces using the PowerSoil kit (MoBio, USA), according to the manufacturer's instructions.

\section{Polymerase chain reaction (PCR) amplification of genetic loci}

Genomic DNA samples were subjected to nested PCRbased analyses of three loci. For the specific identification of Cryptosporidium, a portion of the SSU gene ( 240 bp) was used [13], and genotypic/subgenotypic classification was achieved employing part of the gp60 gene (250$350 \mathrm{bp}$ ) [13]. For the genetic characterisation of Giardia (to the level of assemblage), a portion of the tpi gene ( $\sim 530 \mathrm{bp})$ was employed [21]. PCR was carried out in a volume of 


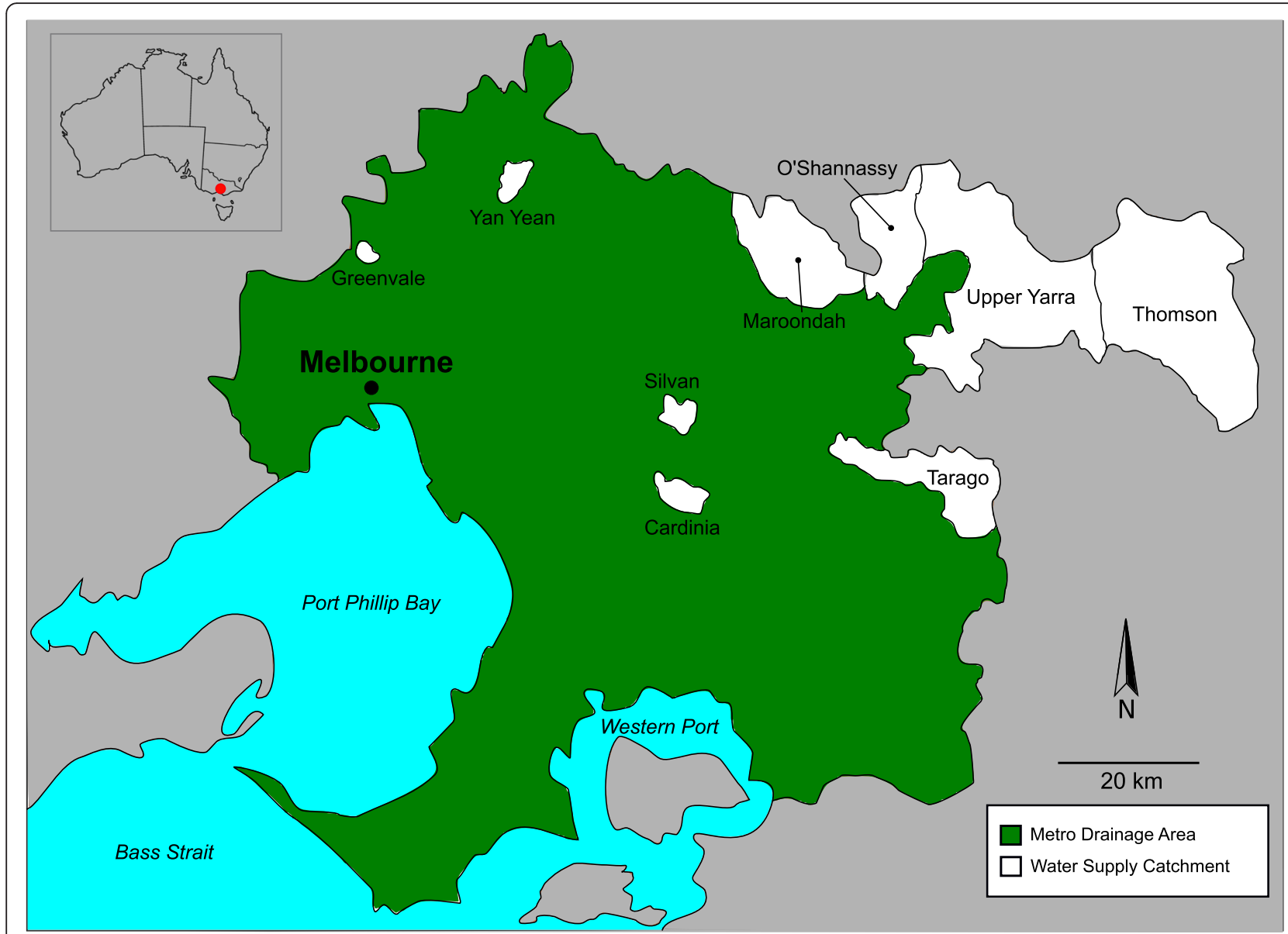

Fig. 1 Map of the Melbourne Water catchment areas from where the 4,256 faecal samples from animals were collected

$50 \mu \mathrm{l}$ containing $10 \mathrm{mM}$ Tris- $\mathrm{HCl}(\mathrm{pH} 8.4), 50 \mathrm{mM} \mathrm{KCl}$ (Promega, Madison, USA), $2.0-3.0 \mathrm{mM}$ of $\mathrm{MgCl}_{2}$ (depending on the locus), $200 \mu \mathrm{M}$ of each deoxynucleotide triphosphate, $50 \mathrm{pmol}$ of each primer and $1 \mathrm{U}$ of either GoTaq (Promega) or MangoTa $q^{\text {tim }}$ (Bioline, USA) DNA polymerase.

For Cryptosporidium, primary amplification of SSU was achieved using primers XF2 (forward: 5'-GGA AGG GTT GTA TTT ATT AGA TAA AG-3') and XR2 (reverse: 5'AAG GAG TAA GGA ACA ACC TCC A-3') [22], followed by nested amplification of SSU using the internal primers pSSUf (forward: 5'-AAA GCT CGT AGT TGG ATT TCT GTT-3') and pSSUr (reverse: 5'-ACC TCT GAC TGT TAA ATA CRA ATG C-3') [23]. For primary amplification, a cycling protocol of $94{ }^{\circ} \mathrm{C}$ for $5 \mathrm{~min}$ (initial denaturation), followed by 30 cycles of $94{ }^{\circ} \mathrm{C}$ for $45 \mathrm{~s}$ (denaturation), $45^{\circ} \mathrm{C}$ for $2 \mathrm{~min}$ (annealing) and $72{ }^{\circ} \mathrm{C}$ for $1.5 \mathrm{~min}$ (extension), with a final extension of $72{ }^{\circ} \mathrm{C}$ for $10 \mathrm{~min}$ was employed. Secondary amplification was achieved employing $94{ }^{\circ} \mathrm{C}$ for 5 min, followed by 35 cycles of $94{ }^{\circ} \mathrm{C}$ for $30 \mathrm{~s}, 55^{\circ} \mathrm{C}$ for $30 \mathrm{~s}$, and $72{ }^{\circ} \mathrm{C}$ for $30 \mathrm{~s}$, with a final extension of $72{ }^{\circ} \mathrm{C}$ for $10 \mathrm{~min}$.
For selected samples, Cryptosporidium was further characterized using a longer region ( $590 \mathrm{bp})$ of the $S S U$ gene. This region was first PCR-amplified employing primers 18SiCF2 (forward: 5'-GAC ATA TCA TTC AAG TTT CTG ACC-3') and 18SiCR2 (reverse: 5'-CTG AAG GAG TAA GGA ACA ACC-3'), followed by a nested amplification using primers $18 \mathrm{SiCF} 1$ (forward: 5'-CCT ATC AGC TTT AGA CGG TAG G-3') and 18SiCR1 (reverse: 5'-TCT AAG AAT TTC ACC TCT GAC TG-3') [24]. Both amplifications utilized the cycling protocol: $94^{\circ}$ for $5 \mathrm{~min}$ (initial denaturation), followed by 45 cycles of $94{ }^{\circ} \mathrm{C}$ for $30 \mathrm{~s}$ (denaturation), $58{ }^{\circ} \mathrm{C}$ for $30 \mathrm{~s}$ (annealing) and $72{ }^{\circ} \mathrm{C}$ for $30 \mathrm{~s}$ (extension), with a final extension of $72{ }^{\circ} \mathrm{C}$ for $10 \mathrm{~min}$

The $g p 60$ gene $(\sim 1 \mathrm{~kb})$ was first amplified using primers gp15-ATG (forward: 5'-ATG AGA TTG TCG CCT CAT TAT C-3') and gp15-STOP (reverse: 5'-TTA CAA CAC GAA TAA GGC TGC-3') [25], followed by the nested amplification of gp60 using primers gp15-15A (forward: 5'-GCC GTT CCA CTC AGA GGA AC-3') and gp15-15E (reverse: 5'-CCA CAT TAC AAA TGA AGT GCC GC-3') 
[26]. Primary amplification of gp60 utilised the cycling protocol, $94{ }^{\circ} \mathrm{C}$ for $5 \mathrm{~min}$ (initial denaturation), followed by 35 cycles of $94{ }^{\circ} \mathrm{C}$ for $30 \mathrm{~s}$ (denaturation), $55^{\circ} \mathrm{C}$ for $45 \mathrm{~s}$ (annealing) and $72{ }^{\circ} \mathrm{C}$ for $1 \mathrm{~min}$ (extension), with a final extension of $72{ }^{\circ} \mathrm{C}$ for $10 \mathrm{~min}$. For the amplification of gp60, we employed $94{ }^{\circ} \mathrm{C}$ for $5 \mathrm{~min}$, followed by 30 cycles of $94{ }^{\circ} \mathrm{C}$ for $30 \mathrm{~s}, 55^{\circ} \mathrm{C}$ for $30 \mathrm{~s}$, and $72{ }^{\circ} \mathrm{C}$ for $30 \mathrm{~s}$, with a final extension at $72{ }^{\circ} \mathrm{C}$ for $10 \mathrm{~min}$.

For some samples, Cryptosporidium was further characterized using a longer region ( $~ 850 \mathrm{bp})$ of the gp60 gene. This region was PCR-amplified using primers AL3531 (forward: 5'-ATA GTC TCC GCT GTA TTC-3') and AL3535 (reverse: 5'-GGA AGG AAC GAT GTA TCT-3'), followed by a nested amplification using primers AL3532 (forward: 5'-TCC GCT GTA TTC TCA GCC-3') and AL3534 (reverse: 5'-GCA GAG GAA CCA GCA TC-3') [27]. For both amplifications, the following cycling protocol was used: $94{ }^{\circ} \mathrm{C}$ for 5 min (initial denaturation), followed by 35 cycles of $94{ }^{\circ} \mathrm{C}$ for $45 \mathrm{~s}$ (denaturation), $50{ }^{\circ} \mathrm{C}$ for $45 \mathrm{~s}$ (annealing) and $72{ }^{\circ} \mathrm{C}$ for $60 \mathrm{~s}$ (extension), with a final extension of $72{ }^{\circ} \mathrm{C}$ for $10 \mathrm{~min}$.

For Giardia, the tpi locus was amplified using primers AL3543 (forward: 5'-AAA TTA TGC CTG CTC GTC G-3') and AL3546 (reverse: 5'-CAA ACC TTT TCC GCA AAC C-3'), followed by the nested amplification of tpi employing primers AL3544 (forward: 5'-CCC TTC ATC GGT GGT AAC TT-3') and AL3545 (reverse: 5'-GTG GCC ACC ACT CCC GTG CC-3') [21]. For the primary amplification, the cycling protocol was $94{ }^{\circ} \mathrm{C}$ for $5 \mathrm{~min}$ (initial denaturation), followed by 35 cycles of $94{ }^{\circ} \mathrm{C}$ for $45 \mathrm{~s}$ (denaturation), $50{ }^{\circ} \mathrm{C}$ for $45 \mathrm{~s}$ (annealing), and $72{ }^{\circ} \mathrm{C}$ for $1 \mathrm{~min}$ (extension) and a final extension of $72{ }^{\circ} \mathrm{C}$ for $10 \mathrm{~min}$. Secondary amplification of tpi was achieved employing $94{ }^{\circ} \mathrm{C}$ for $5 \mathrm{~min}$, followed by 35 cycles of $94{ }^{\circ} \mathrm{C}$ for $45 \mathrm{~s}, 55{ }^{\circ} \mathrm{C}$ for $30 \mathrm{~s}$, and $72{ }^{\circ} \mathrm{C}$ for $1 \mathrm{~min}$, with a final extension at $72{ }^{\circ} \mathrm{C}$ for $10 \mathrm{~min}$.

\section{Mutation scanning, sequencing and phylogenetic analyses} Single-strand conformation polymorphism (SSCP) analysis was used to scan for sequence variation within and among SSU and gp60 amplicons (e.g. [13, 16]). In brief, $1 \mu \mathrm{l}$ of each secondary amplicon (<450 bp) was mixed with $5 \mu \mathrm{l}$ of DNA sequencing-stop solution (Promega) and $5 \mu \mathrm{l}$ of $\mathrm{H}_{2} \mathrm{O}$, heat-denatured at $94{ }^{\circ} \mathrm{C} / 30 \mathrm{~min}$, snapcooled on a freeze-block $\left(-20^{\circ} \mathrm{C}\right)$ and then subjected to electrophoresis at $74 \mathrm{~V}$ at $7.4{ }^{\circ} \mathrm{C}$ (constant) for $16 \mathrm{~h}$ in a GMA Wide Mini S-2x25 gel in a SEA 2000 rig (Elchrom Scientific AG) using TAE buffer (40 mM Tris base, $20 \mathrm{mM}$ acetic acid, 1.0 mM EDTA, Bio-Rad, USA). A control sample (representing a known genotype) was included on each gel to ensure the reproducibility of profiles representing this sample among gels.

Following SSCP-based analysis, selected amplicons representing each distinct electrophoretic profile and all SSU, gp60 (Cryptosporidium) and tpi (Giardia) amplicons were treated with shrimp alkaline phosphatase and exonuclease I (ThermoFisher, Waltham, USA), according to the manufacturer's instructions, and then subjected to bi-directional automated sequencing (BigDye ${ }^{\circ}$ Terminator v.3.1 chemistry, Applied Biosystems, USA) using the same primers employed in the secondary PCR. Sequence quality was verified by comparison with corresponding electropherograms using the program Geneious v.8 [28]. Sequences were aligned using the program MUSCLE [29], and alignments were adjusted manually using the program Mesquite v.2.75 [30]. Sequences were then compared with those available in the GenBank database using BLASTn.

Phylogenetic analysis of sequence data was conducted by Bayesian inference (BI) using Monte Carlo Markov Chain (MCMC) analysis in MrBayes v.3.2.3 [31]. The likelihood parameters set for BI analysis of SSU data were based on the Akaike Information Criteria (AIC) test in jModeltest v.2.1.7 [32]. For SSU (Cryptosporidium) and tpi (Giardia) data, the number of substitutions (Nst) was set at 6, with a gamma-distribution and a proportion of invariable sites. For the separate analyses of gp60 (Cryptosporidium) sequence data, the Nst was set at 6, with an equal rate among sites. Posterior probability (pp) values were calculated by running 2,000,000 generations with four simultaneous tree-building chains. Trees were saved every 100th generation. At the end of each run, the standard deviation of split frequencies was $<0.01$, and the potential scale reduction factor approached one. A $50 \%$ majority rule consensus tree for each analysis was constructed based on the final $75 \%$ of trees generated by BI. Analyses were run three times to ensure convergence and insensitivity to priors. Outgroups used in the analyses were Giardia muris for G. duodenalis (tpi), C. hominis for gp60 and C. muris for SSU.

\section{Results \\ Molecular detection of Cryptosporidium, and taxon identity based on SSU}

We conducted mutation scanning and sequence analyses of all amplicons $(n=69)$ produced from 4,256 $(1.62 \%)$ faecal DNA samples and identified them to species and/ or genotype of Cryptosporidium. A total of 64 samples were characterised by their SSU sequences; 52 were assigned GenBank accession nos. (KU531647-KU531698; Tables 1 and 2), of which 24 sequences were selected as representatives for phylogenetic analysis (Fig. 2; Additional file 2: Table S4). In total, there were 12 novel sequences (i.e. $<100 \%$ identity with a sequence on GenBank) for $S S U$. Samples that were test-positive for SSU were assessed according to catchment (Additional file 1: Table S2) and host (Additional file 1: Table S3). Overall, prevalence was assessed by catchment in Table 2 . 
Table 1 Summary of epidemiological and molecular information pertaining to the pathogen test-positive faecal samples collected from the Melbourne Water catchments (July 2011 to November 2015)

\begin{tabular}{|c|c|c|c|c|c|c|c|}
\hline Sample code & Host & Locality & Date & Pathogen & Typing & Method & $\begin{array}{l}\text { GenBank } \\
\text { accession no. }\end{array}$ \\
\hline MR4158 & Wombat & Maroondah & 12-Dec-13 & Cryptosporidium fayeri & SSU & Sequencing & KU531671 \\
\hline MR4198 & Wombat & Maroondah & 12-Dec-13 & Cryptosporidium fayeri & SSU & SSCP & KU531671 ${ }^{\mathrm{a}}$ \\
\hline MR4199 & Wombat & Maroondah & 12-Dec-13 & Cryptosporidium fayeri & SSU & SSCP & KU531671 ${ }^{\mathrm{a}}$ \\
\hline MR4200 & Wombat & Maroondah & 12-Dec-13 & Cryptosporidium fayeri & SSU & SSCP & KU531671 ${ }^{\mathrm{a}}$ \\
\hline MR4211 & Wombat & Maroondah & 12-Dec-13 & Cryptosporidium fayeri & SSU & SSCP & KU531671 ${ }^{\mathrm{a}}$ \\
\hline MR4231 & Wombat & Maroondah & 12-Dec-13 & Cryptosporidium fayeri & SSU & Sequencing & KU531672 \\
\hline GV3073 & Kangaroo & Greenvale & $16-O c t-12$ & Cryptosporidium fayeri-like & SSU & Sequencing & KU531656 \\
\hline YY3126 & Kangaroo & Yan Yean & $16-O c t-12$ & Cryptosporidium fayeri-like & SSU & Sequencing & KU531658 \\
\hline C3616 & Wombat & Cardinia & 31-Jul-13 & Cryptosporidium fayeri-like & SSU & Sequencing & KU531666 \\
\hline YY6091 & Kangaroo & Yan Yean & 09-Jul-15 & Cryptosporidium sp. EGK1 genotype (C. fayeri-like) & SSU & Sequencing & KU531695 \\
\hline YY6016 & Kangaroo & Yan Yean & 09-Jul-15 & Cryptosporidium sp. Kangaroo genotype I (C. fayeri-like) & SSU & Sequencing & KU531694 \\
\hline OS2785 & Deer & O'Shannassy & Jul-12 & Cryptosporidium hominis & SSU & Sequencing & na \\
\hline UY3513 & Deer & Upper Yarra & 25-Jun-13 & Cryptosporidium hominis & SSU & Sequencing & KU531663 \\
\hline MR3443 & Wallaby & Maroondah & 09-May-13 & Cryptosporidium hominis (IBA10G2) & gp60 & Sequencing & KU531699 \\
\hline GV3952 & Kangaroo & Greenvale & 20-Aug-13 & Cryptosporidium parvum & SSU & Sequencing & KU531669 \\
\hline SV5306 & Rabbit & Silvan & 26-Nov-14 & Cryptosporidium cuniculus & SSU & Sequencing & KU531683 \\
\hline GV6100 & Rabbit & Greenvale & 09-Jul-15 & Cryptosporidium cuniculus (VbA24) & SSU & SSCP & KU531697 \\
\hline GV6100 & Rabbit & Greenvale & 09-Jul-15 & Cryptosporidium cuniculus (VbA24) & gp60 & Sequencing & KU531704 \\
\hline SV5945 & Rabbit & Silvan & 21-May-15 & Cryptosporidium cuniculus (VbA25) & SSU & Sequencing & KU531693 \\
\hline SV5945 & Rabbit & Silvan & 21-May-15 & Cryptosporidium cuniculus (VbA25) & gp60 & Sequencing & KU531702 \\
\hline GV6098 & Rabbit & Greenvale & 09-Jul-15 & Cryptosporidium cuniculus (VbA25) & SSU & Sequencing & KU531696 \\
\hline GV6098 & Rabbit & Greenvale & 09-Jul-15 & Cryptosporidium cuniculus (VbA25) & gp60 & Sequencing & KU531703 \\
\hline YY3790 & Kangaroo & Yan Yean & 20-Aug-13 & Cryptosporidium cuniculus (VbA26) & gp60 & Sequencing & KM366140 \\
\hline YY3790 & Kangaroo & Yan Yean & 20-Aug-13 & Cryptosporidium cuniculus (VbA26) & SSU & Sequencing & KM366142 \\
\hline YY3809 & Kangaroo & Yan Yean & 20-Aug-13 & Cryptosporidium cuniculus (VbA26) & gp60 & Sequencing & KU531700 \\
\hline GV5010 & Rabbit & Greenvale & 04-Sep-14 & Cryptosporidium cuniculus (VbA26) & gp60 & Sequencing & KU531701 \\
\hline GV6131 & Rabbit & Greenvale & 09-Jul-15 & Cryptosporidium cuniculus (VbA26) & gp60 & SSCP & KU531705 \\
\hline GV6132 & Rabbit & Greenvale & 09-Jul-15 & Cryptosporidium cuniculus (VbA26) & gp60 & SSCP & KU531705 \\
\hline GV6137 & Rabbit & Greenvale & 09-Jul-15 & Cryptosporidium cuniculus (VbA26) & SSU & Sequencing & KU531697 \\
\hline GV6137 & Rabbit & Greenvale & 09-Jul-15 & Cryptosporidium cuniculus (VbA26) & gp60 & Sequencing & KU531705 \\
\hline C5371 & Emu & Cardinia & 08-Jan-15 & Cryptosporidium canis & SSU & Sequencing & KU531684 \\
\hline OS3311 & Deer & O'Shannassay & 08-Apr-13 & Cryptosporidium suis-like & SSU & Sequencing & KU531660 \\
\hline C2202 & Deer & Cardinia & Aug-11 & Cryptosporidium ubiquitum & SSU & Sequencing & KU531647 \\
\hline OS5301 & Deer & O'Shannassay & $26-N o v-14$ & Cryptosporidium ubiquitum & SSU & Sequencing & KU531682 \\
\hline OS6339 & Deer & O'Shannassay & 20-Nov-15 & Cryptosporidium ubiquitum & SSU & Sequencing & KU531698 \\
\hline C3604 & Wombat & Cardinia & 31-Jul-13 & Cryptosporidium ubiquitum-like & SSU & Sequencing & KU531665 \\
\hline OS5267 & Wombat & O'Shannassay & 26-Nov-14 & Cryptosporidium ubiquitum-like & SSU & Sequencing & KU531681 \\
\hline GV3044 & Kangaroo & Greenvale & $16-O c t-12$ & Cryptosporidium macropodum & SSU & Sequencing & KU531655 \\
\hline GV4434 & Kangaroo & Greenvale & 14-Mar-14 & Cryptosporidium macropodum & SSU & Sequencing & KU531673 \\
\hline GV4441 & Kangaroo & Greenvale & 14-Mar-14 & Cryptosporidium macropodum & SSU & Sequencing & KU531674 \\
\hline GV4992 & Kangaroo & Greenvale & 04-Sep-14 & Cryptosporidium macropodum & SSU & Sequencing & KU531677 \\
\hline GV4994 & Kangaroo & Greenvale & 04-Sep-14 & Cryptosporidium macropodum & SSU & SSCP & KU531677 \\
\hline GV5000 & Kangaroo & Greenvale & 04-Sep-14 & Cryptosporidium macropodum & SSU & SSCP & KU531677 \\
\hline YY5091 & Kangaroo & Yan Yean & 04-Sep-14 & Cryptosporidium macropodum & SSU & Sequencing & KU531678 \\
\hline
\end{tabular}


Table 1 Summary of epidemiological and molecular information pertaining to the pathogen test-positive faecal samples collected from the Melbourne Water catchments (July 2011 to November 2015) (Continued)

\begin{tabular}{|c|c|c|c|c|c|c|c|}
\hline GV5505 & Kangaroo & Greenvale & 20-Feb-15 & Cryptosporidium macropodum & SSU & Sequencing & KU531685 \\
\hline GV5543 & Kangaroo & Greenvale & 20-Feb-15 & Cryptosporidium macropodum & SSU & Sequencing & KU531686 \\
\hline GV5552 & Kangaroo & Greenvale & 20-Feb-15 & Cryptosporidium macropodum & SSU & Sequencing & KU531687 \\
\hline GV5558 & Kangaroo & Greenvale & 20-Feb-15 & Cryptosporidium macropodum & SSU & SSCP & KU531688 \\
\hline GV5563 & Kangaroo & Greenvale & 20-Feb-15 & Cryptosporidium macropodum & SSU & SSCP & KU531688 \\
\hline GV5573 & Kangaroo & Greenvale & 20-Feb-15 & Cryptosporidium macropodum & SSU & Sequencing & KU531688 \\
\hline OS2816 & Wallaby & O'Shannassy & Jul-12 & Cryptosporidium macropodum-like & SSU & Sequencing & KU531649 \\
\hline OS2827 & Wallaby & O'Shannassy & Jul-12 & Cryptosporidium macropodum-like & SSU & Sequencing & KU531652 \\
\hline SV3188 & Wallaby & Silvan & 19-Nov-12 & Cryptosporidium macropodum-like & SSU & Sequencing & KU531659 \\
\hline OS3365 & Wallaby & O'Shannassay & 08-Apr-13 & Cryptosporidium macropodum-like & SSU & Sequencing & KU531661 \\
\hline C 3623 & Wallaby & Cardinia & 31-Jul-13 & Cryptosporidium macropodum-like & SSU & Sequencing & KU531667 \\
\hline OS5235 & Wallaby & O'Shannassay & 26-Nov-14 & Cryptosporidium macropodum-like & SSU & Sequencing & KU531679 \\
\hline UY5645 & Waterbird & Upper Yarra & 30-Mar-15 & Cryptosporidium sp. duck genotype-like & SSU & Sequencing & KU531689 \\
\hline UY5649 & Waterbird & Upper Yarra & 30-Mar-15 & Cryptosporidium sp. duck genotype-like & SSU & SSCP & KU531689 \\
\hline UY2975 & Waterbird & Upper Yarra & 30-Aug-12 & Cryptosporidium sp. duck-like genotype & SSU & Sequencing & KU531654 \\
\hline OS4106 & Deer & O'Shannassay & 19-Nov-13 & Cryptosporidium ryanae-like MW2 & SSU & Sequencing & KU531670 \\
\hline OS5242 & Deer & O'Shannassay & 26-Nov-14 & Cryptosporidium ryanae-like MW2 & SSU & Sequencing & KU531680 \\
\hline C5875 & Deer & Cardinia & 21-May-15 & Cryptosporidium ryanae-like MW2 & SSU & Sequencing & KU531692 \\
\hline OS2316 & Deer & O'Shannassy & Dec-11 & Cryptosporidium ryanae-like MW4 & SSU & Sequencing & KU531648 \\
\hline YY3874 & Deer & Yan Yean & 20-Aug-13 & Cryptosporidium ryanae-like MW4 & SSU & Sequencing & KU531668 \\
\hline OS4606 & Deer & O'Shannassay & 14-Apr-14 & Cryptosporidium ryanae-like MW4 & SSU & Sequencing & KU531675 \\
\hline C4873 & Deer & Cardinia & 24-Jul-14 & Cryptosporidium ryanae-like MW4 & SSU & Sequencing & KU531676 \\
\hline UY5700 & Deer & Upper Yarra & 30-Mar-15 & Cryptosporidium ryanae-like MW4 & SSU & Sequencing & KU531690 \\
\hline C5846 & Deer & Cardinia & 21-May-15 & Cryptosporidium ryanae-like MW4 & SSU & Sequencing & KU531691 \\
\hline OS2821 & Deer & O'Shannassy & Jul-12 & Cryptosporidium ryanae-like MW7 & SSU & Sequencing & KU531650 \\
\hline OS2822 & Deer & O'Shannassy & Jul-12 & Cryptosporidium ryanae-like MW7 & SSU & Sequencing & KU531651 \\
\hline MR3424 & Deer & Maroondah & 09-May-13 & Cryptosporidium ryanae-like MW7 & SSU & Sequencing & KU531662 \\
\hline UY2900 & Deer & Upper Yarra & 30-Aug-12 & Cryptosporidium sp. deer genotype & SSU & Sequencing & KU531653 \\
\hline YY3101 & Deer & Yan Yean & $16-O c t-12$ & Cryptosporidium sp. deer genotype & SSU & Sequencing & KU531657 \\
\hline UY3518 & Deer & Upper Yarra & 25-Jun-13 & Cryptosporidium sp. deer genotype & SSU & Sequencing & KU531664 \\
\hline $\mathrm{TH} 2278$ & Rabbit & Thomson & 25-Sep-11 & Giardia duodenalis Al & tpi & Sequencing & KU531708 \\
\hline TH2291 & Deer & Thomson & 25-Sep-11 & Giardia duodenalis Al & tpi & Sequencing & KU531709 \\
\hline SV2382 & Kangaroo & Silvan & 2-Dec-11 & Giardia duodenalis Al & tpi & Sequencing & KU531710 \\
\hline MR4752 & Wombat & Maroondah & 18-Jun-14 & Giardia duodenalis Al & tpi & Sequencing & KU531718 \\
\hline TAR2129 & Deer & Tarago & 07-Jul-11 & Giardia duodenalis Alll & $t p i$ & Sequencing & KU531706 \\
\hline TAR2135 & Deer & Tarago & 07-Jul-11 & Giardia duodenalis Alll & tpi & Sequencing & KU531707 \\
\hline OS4115 & Deer & O'Shannassy & 19-Nov-13 & Giardia duodenalis Alll & tpi & Sequencing & KU531711 \\
\hline OS4135 & Deer & O'Shannassy & 19-Nov-13 & Giardia duodenalis Alll & tpi & Sequencing & KU531712 \\
\hline UY4624 & Deer & Upper Yarra & 20-May-14 & Giardia duodenalis Alll & tpi & Sequencing & KU531713 \\
\hline UY4634 & Deer & Upper Yarra & 20-May-14 & Giardia duodenalis Alll & tpi & Sequencing & KU531714 \\
\hline UY4635 & Deer & Upper Yarra & 20-May-14 & Giardia duodenalis Alll & tpi & Sequencing & KU531715 \\
\hline UY4638 & Deer & Upper Yarra & 20-May-14 & Giardia duodenalis Alll & tpi & Sequencing & KU531716 \\
\hline UY4661 & Deer & Upper Yarra & 20-May-14 & Giardia duodenalis Alll & tpi & Sequencing & KU531717 \\
\hline
\end{tabular}

Bold-type indicates a novel genotype. na: not available; length of the sequence determined $(<200 \mathrm{bp})$ was less than that required to be assigned a GenBank accession number; sequence available from authors. ${ }^{\text {a }}$ indicates accession number represented by an SSCP profile 
Table 2 The total numbers of each host sampled in each catchment, as part of the Melbourne Water Corporation sampling program for waterborne pathogens (July 2011 to November 2015), together with the numbers of animals PCR test-positive for species/genotypes of Cryptosporidium (number of test-positive samples)

\begin{tabular}{|c|c|c|c|c|c|c|c|c|c|}
\hline Catchment & Emu & Waterbird & Deer & Rabbit & Kangaroo & Wallaby & Wombat & Total & Prevalence (\%) \\
\hline Cardinia & $\begin{array}{l}1 C . \\
\text { canis }\end{array}$ & & $\begin{array}{l}4 \text { (3 C. ryanae, } \\
1 \text { C. ubiquitum) }\end{array}$ & & & $\begin{array}{l}1 \mathrm{C} . \\
\text { macropodum }\end{array}$ & $\begin{array}{l}2 \text { (1 C. fayeri, } \mathbf{1} \\
\text { C. ubiquitum) }\end{array}$ & 718 & 1.11 \\
\hline Greenvale & & & & $\begin{array}{l}6 C . \\
\text { cuniculus }\end{array}$ & $\begin{array}{l}\text { 14 (12 C. macropodum, } \\
\text { 1. C. fayeri, } 1 \text { C. parvum) }\end{array}$ & & & 638 & 3.13 \\
\hline Maroondah & & & 1 C. ryanae & & & 1 C. hominis & 6 C. fayeri & 344 & 2.32 \\
\hline O'Shannassay & & & $\begin{array}{l}9 \text { (6 C. ryanae, } 1 \text { C. } \\
\text { hominis, } 1 \text { C. suis, } \\
1 \text { C. ubiquitum) }\end{array}$ & & 1 C. macropodum & $\begin{array}{l}3 \mathrm{C} \text {. } \\
\text { macropodum }\end{array}$ & $\begin{array}{l}1 \mathrm{C} . \\
\text { ubiquitum }\end{array}$ & 622 & 2.25 \\
\hline Silvan & & & & $\begin{array}{l}2 \mathrm{C} . \\
\text { cuniculus }\end{array}$ & & $\begin{array}{l}1 \mathrm{C} . \\
\text { macropodum }\end{array}$ & & 527 & 0.57 \\
\hline Tarago & & & & & & & & 61 & 0 \\
\hline Thomson & & & & & & & & 31 & 0 \\
\hline Upper Yarra & & $\begin{array}{l}3 \text { C. sp. duck } \\
\text { genotype }\end{array}$ & $\begin{array}{l}4 \text { (3 C. ryanae, } \\
1 \text { C. hominis) }\end{array}$ & & & & & 714 & 0.98 \\
\hline Yan Yean & & & 2 C. ryanae & & $\begin{array}{l}6 \text { (2 C. cuniculus, } 2 \text { C. sp. } \\
\text { Kangaroo genotype, } 1 \text { C. } \\
\text { macropodum, } 1 \text { C. fayeri) }\end{array}$ & & & 601 & 1.33 \\
\hline
\end{tabular}

Each positive sample is identified to the nearest major Cryptosporidium clade. Overall prevalence for Cryptosporidium was $1.6 \%$. Species commonly reported in humans (26\%) are in bold-type. Bird, Reptile, Cat, Dog, Fox, Rat, Possum and Unknown groups were all test-negative for Cryptosporidium

In the following list, we assign individual taxa identified in the present study (based on the sequencing of $S S U)$ to the most closely related species of Cryptosporidium in particular clades based on sequence identity:

- Members of the C. fayeri clade: Six of 74 (8.10 \%, catchment MR) samples from wallabies were test-positive for C. fayeri. Two of 287 $(0.70 \%, \mathrm{YY})$ and one of $603(0.17 \%, \mathrm{GV})$ samples from kangaroos were test-positive for Cryptosporidium sp. EGK1 (eastern grey kangaroo type 1), two of which were novel genotypes (GenBank accession nos. KU531656 and KU531666). One of 76 (1.32 \%) samples from wallabies from catchment CA was a novel genotype that was similar to $C$. fayeri (KU531658). One of the 287 (0.35\%) samples from kangaroos from catchment $Y Y$ was a novel genotype similar to Cryptosporidium sp. kangaroo genotype I (KU531694). For the first time, C. fayeri and C. fayeri-like genotypes were reported from wombats.

- Members of the C. hominis, C. parvum and C. cuniculus clade:

One of 536 (0.19\%, UY) and one of 408 (0.25\%, OS) from deer were test-positive for $C$. hominis. One of $603(0.17 \%)$ samples from a kangaroo from catchment GV was test-positive for C. parvum. Five of 26 (19.2\%, GV), one of eight (12.5\%, SV) samples and one of 287 $(0.35 \%, \mathrm{YY})$ samples from rabbits were test-positive for C. cuniculus.

- Members of the C. canis clade:
One of $52(1.92 \%)$ samples from emus from catchment CA was test-positive for C. canis.

- Members of the C. suis clade:

One of $536(0.19 \%)$ samples from deer from catchment UY was test-positive for the novel $C$. suis-like genotype (KU531660).

- Members of the C. ubiquitum clade: Two of 408 (0.49 \%) and one of 247 (0.40\%) samples from deer from catchments OS and CA, respectively, were test-positive for $C$. ubiquitum. Two samples from wombats were test-positive for a novel C. ubiquitum-like genotype.

- Members of the C. macropodum clade: Twelve of 603 (1.99\%) and one of 287 (0.35\%) of kangaroos from GV and YY, respectively, were testpositive for C. macropodum. Four of 53 (7.55 \%) samples from wallabies from catchment OS were test-positive for C. macropodum-like genotypes, three of which were novel (KU531649, KU531661 and KU531679). One of 168 (0.60 \%) samples from wallabies from catchment SV were test-positive for a C. macropodum-like genotype.

- Members of the Cryptosporidium sp. duck genotype clade:

Two novel genotypes were identified from three of 55 (5.45 \%) samples from waterbirds from catchment UY (KU531654 and KU531689).

- Members of the C. ryanae clade:

Two of $536(0.37 \%)$ and one of $250(0.40 \%)$ samples from deer were test-positive for Cryptosporidium sp. 


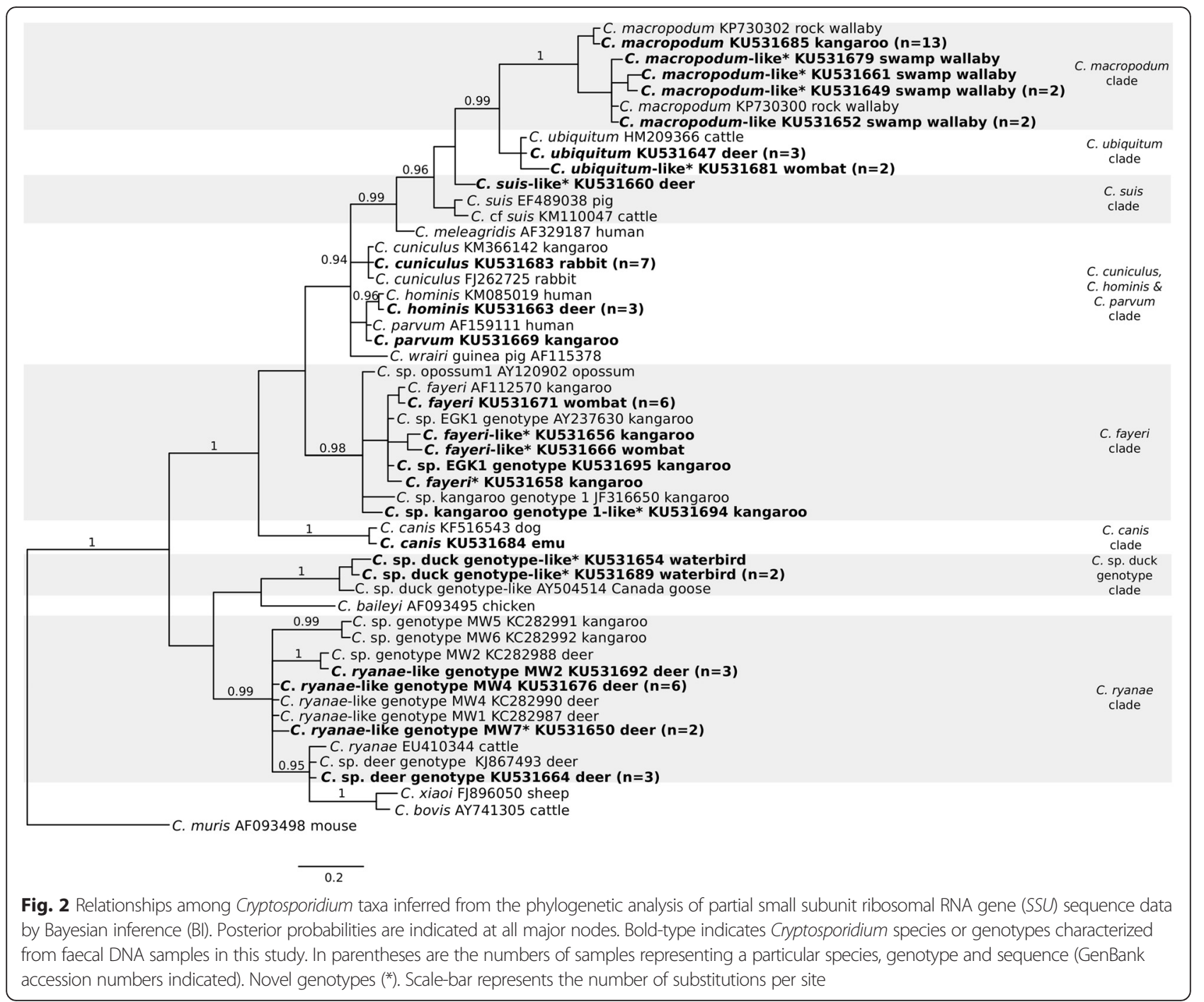

deer genotype from the catchments UY and YY, respectively. One of $536(0.19 \%, \mathrm{UY})$, two of 408 $(0.49 \%$, OS), two of 247 (0.81\%, CA) and one of 250 $(0.40 \%, \mathrm{YY})$ samples from deer were test-positive for the C. ryanae-like MW4 genotype. Two of 408 $(0.49 \%)$ samples from deer from catchment OS were test-positive for the novel genotype $C$. ryanaelike MW7 (KU531650). Two of 408 (0.49\%, OS) and one of $247(0.40 \%, C A)$ samples from deer were test-positive for the C. ryanae-like MW2 genotype.

\section{Cryptosporidium subgenotypes based on gp60}

All ten samples test-positive in PCR for $\operatorname{gp} 60 \quad(n=10)$ were characterised to the level of subgenotype, and seven of them were assigned GenBank accession nos. (KU531699-KU531705). Based on a comparison with reference sequences from GenBank, six unique gp60 sequence types were characterised as $C$. hominis (genotype Ib - subgenotype IbA10G2), and C. cuniculus (genotype $\mathrm{Vb}$ - subgenotype VbA25 and VbA26) (Fig. 3; Table 1 and Additional file 2: Table S5). One of 74 (1.35 \%) samples from wallabies from catchment MR was test-positive for $C$. hominis IbA10G2 (accession no. KJ506839). In addition, Cryptosporidium cuniculus subgenotypes $\mathrm{VbA} 25$ and VbA26 were identified in seven samples from rabbits from catchments GV, YY, SV and MR, four of which were novel. Cryptosporidium cuniculus (VbA26) was identified in two samples from kangaroos from catchment YY (cf. [33]).

\section{Giardia species and assemblages}

Sequencing of all tpi amplicons identified 13 of 4,256 $(0.31 \%)$ individual faecal samples to contain Giardia representing the genetic assemblage A of G. duodenalis, based on direct sequence comparisons. From the 13 samples, we 


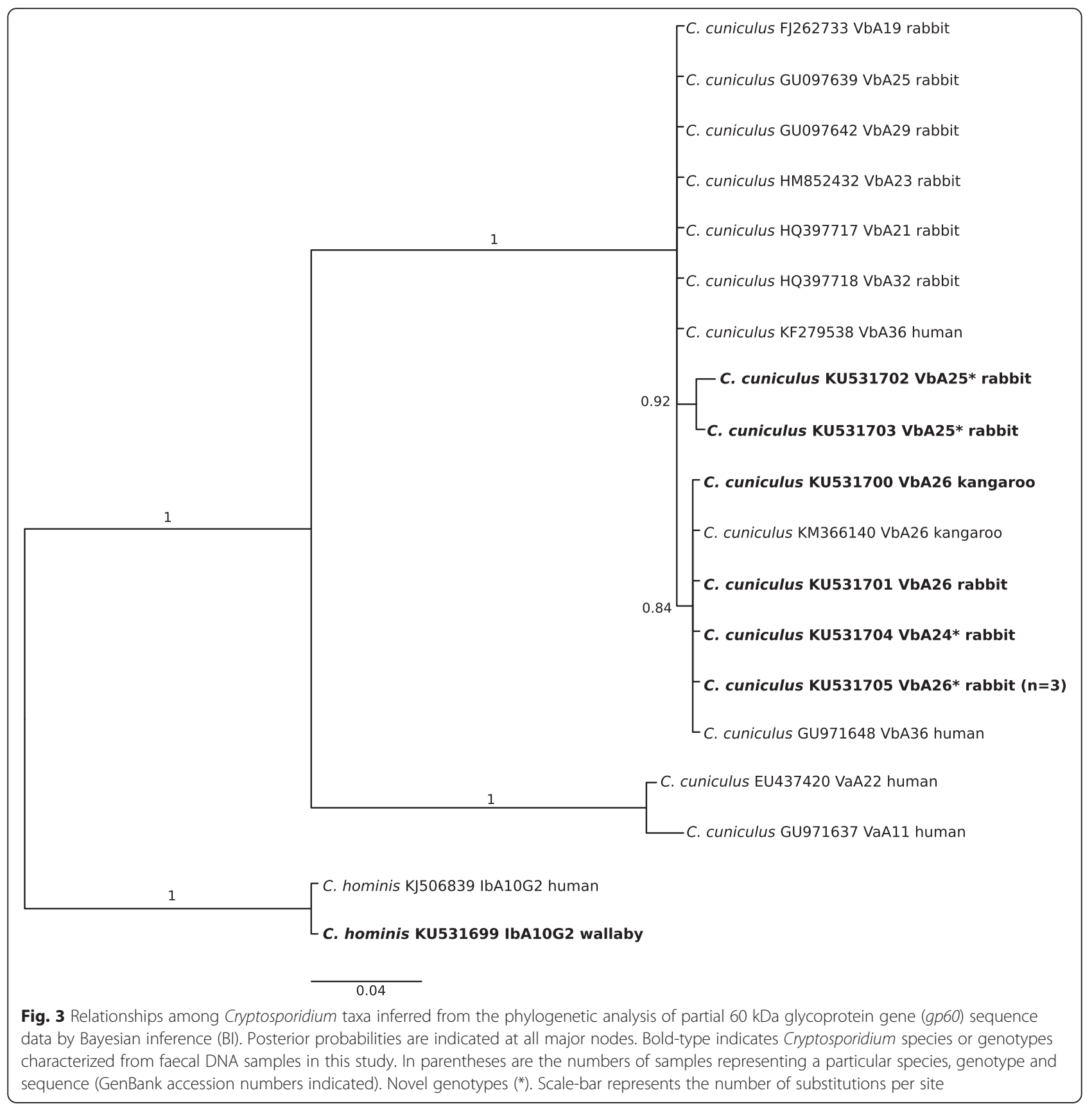

defined three distinct sequence types for tpi (represented by GenBank accession nos. KU531706-KU531718; Fig. 4; Tables 1 and 3 and Additional file 2: Table S6). Giardia sub-assemblage AI was identified in samples from a rabbit and a deer in catchment $\mathrm{TH}$, a wombat in catchment MR and a kangaroo in catchment SV. The genotype (sequence type) of Giardia from deer identified here was novel. Giardia sub-assemblage AIII was identified in nine samples from deer, including five of $536(0.93 \%$, UY), two of 408 $(0.49 \%, \mathrm{OS})$ and two of 18 (11.1\%, TAR) samples from three different catchments.

\section{Discussion}

Extending our initial monitoring program [13], the present study provides a unique perspective of the epidemiology of zoonotic protists found in wildlife inhabiting the water catchment areas of a major metropolitan city (Melbourne). Worldwide, there have been very few comprehensive wildlife surveys in catchments or watershed, with a few notable exceptions (e.g. [34-36]). In Australia, Cryptosporidium and/or Giardia have been described from catchment regions in New South Wales [37-42], Queensland [43], Victoria [13, 33, 44] and Western Australia [45]. 


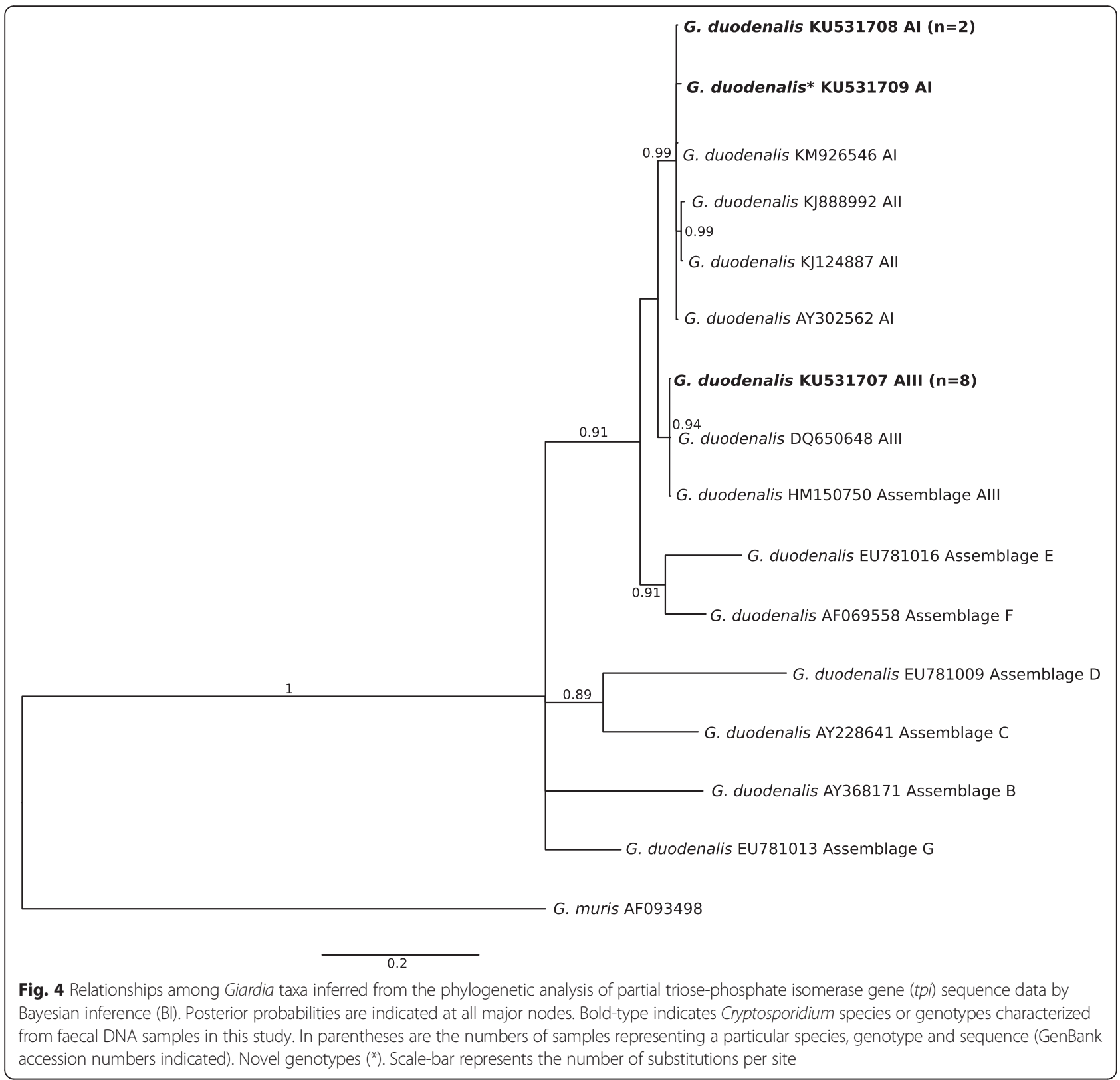

In the first three years of the project (June 2009 to June 2011), 2,009 faecal samples were collected and tested, resulting in a prevalence of $2.8 \%$ for Cryptosporidium and $3.4 \%$ for Giardia [13]. In contrast, over the last four years of the project (July 2011 to November 2015), we tested more than double the number of faecal samples $(n=4,256)$, yet the prevalence of Cryptosporidium and Giardia were $1.62 \%$ and $0.31 \%$, respectively. Overall, the prevalence of these protists was usually less than that reported previously in New South Wales [38, 39, 42], Victoria [13] and Western Australia [45]. The low prevalence recorded here (compared with surveys in other states of Australia) might be a consequence of testing a greater number of samples, the host groups tested, differences in local habitats and/or catchment management practices and/or proximity to agricultural land. Many factors may account for the low prevalence of Cryptosporidium and Giardia in Melbourne's catchments over the six-year duration of the project, including animal culls, changing water levels of the reservoirs and the end of a nine-year drought [46]. One factor influencing the higher prevalence of Giardia in the earlier years (cf. [13]) was a hot-spot event recorded in catchment YY in April 2010, which did not recur the subsequent years. Indeed, no Giardia was found in catchment $\mathrm{YY}$ at any stage during the present investigation. Interestingly, the presence of the ungulate-specific G. duodenalis sub-assemblage AIII in the catchments was only recorded 
Table 3 The total numbers of samples from individual host animals for each catchment, as part of the Melbourne Water Corporation sampling program for waterborne pathogens (July 2011 to November 2015), together with the numbers of animals PCR test-positive for species/genotypes of Giardia (number of test-positive samples)

\begin{tabular}{|c|c|c|c|c|c|c|}
\hline Catchment & Deer & Rabbit & Kangaroo & Wombat & Total & $\begin{array}{l}\text { Prevalence } \\
(\%)\end{array}$ \\
\hline Cardinia & & & & & 718 & 0 \\
\hline Greenvale & & & & & 638 & 0 \\
\hline Maroondah & & & & $1(\mathrm{Al})$ & 344 & 0.29 \\
\hline O'Shannassay & 2 (Alll) & & & & 622 & 0.32 \\
\hline Silvan & & & $1(\mathrm{Al})$ & & 527 & 0.19 \\
\hline Tarago & $2(\mathrm{Al})$ & & & & 61 & 3.28 \\
\hline Thomson & $1(\mathrm{Al})$ & $1(\mathrm{Al})$ & & & 31 & 6.45 \\
\hline Upper Yarra & 5 (Alll) & & & & 714 & 0.70 \\
\hline Yan Yean & & & & & 601 & 0 \\
\hline
\end{tabular}

Sub-assemblage Al is common in wildlife and humans while sub-assemblage All, or fallow deer sub-assemblage, has only been found in deer. Overall prevalence for Giardia was $0.3 \%$. Species commonly reported in humans (46\%) are in bold-type. Bird, Emu, Waterbird, Reptile, Cat, Dog, Fox, Rat, Possum, Wallaby and Unknown groups were all test-negative for Giardia

once between 2009 and 2011, and the first record of Cryptosporidium from a wombat was recorded in 2013, highlighting the rarity and ephemeral nature of these protists in this catchment system.

In the present study, we used a PCR-based approach to genetically characterise 82 samples, which were assigned to seven recognised species of Cryptosporidium (represented by the GenBank accession nos. KU531647-KU531705), and to the genetic assemblage 'AI' and 'AIII' of G. duodenalis (accession nos. KU531706-KU531718). Of the recognised species of Cryptosporidium, only C. macropodum, detected here in kangaroos, has not been reported previously from humans. The remaining six potentially zoonotic species (C. hominis, C. parvum, C. cuniculus, C. ubiquitum, C. canis and C. fayeri) were recorded from deer, emu, kangaroo, rabbit, wallaby and wombat, respectively, and from catchments CA, GV, OS, MR, SV, UY and YY (see Additional file 1: Table S1). Of the 29 species and $<40$ reported genotypes of Cryptosporidium currently recognised [8], the causative agents of human cryptosporidiosis are typically C. hominis or C. parvum (see [47-51]); these parasites have been linked to numerous waterborne outbreaks around the world (reviewed in $[52,53]$ ). Despite the detection of both species in catchments regions surrounding Melbourne, their low prevalence $(0.07 \%$ for $C$. hominis and $0.02 \%$ for $C$. parvum) might suggest a low risk of waterborne transmission to humans. Nonetheless, other species and genotypes of Cryptosporidium may have some zoonotic significance. For example, C. cuniculus, with a prevalence of $0.26 \%$, was implicated in a zoonotic outbreak of cryptosporidiosis in humans in England in 2008
[54] and was linked to a number of sporadic human cases across the UK in 2007 and 2008 [55, 56], and was detected for the first time in a kangaroo in the YY catchment [33]. Cryptosporidium ubiquitum, detected here in deer in catchments CA and UY, might also be a concern, as it has been proposed to present a potential public health risk due to its broad geographical and host ranges, including humans in industrialised nations [49, 57, 58]. It is plausible that $C$. canis $(0.02 \%)$ and C. fayeri $(0.26 \%)$ might also represent minor risks to humans; $C$. canis has been detected in humans around the world [59], and $C$. fayeri was detected in a patient in New South Wales suffering from a prolonged gastrointestinal illness [60].

In the present study, we were able to assign Cryptosporidium to particular clades of species based on sequence identity in SSU, and to genotypes/subgenotypes also based on their gp60 sequence. Within the $C$. fayeri clade, several novel genotypes characterised from samples from kangaroos were all very similar genetically to the marsupialspecific $C$. fayeri. Here, we also report the first molecularly characterized Cryptosporidium genotypes from wombats. To date, there is only one record of Cryptosporidium from a wombat [61]; however, this was not included in the review of Cryptosporidium of marsupials [62]. Wombat faeces have been tested for Cryptosporidium in multiple surveys [13, 39, 42, 63], yielding no test-positive results. Over the six years of our monitoring of Melbourne Water catchment areas ([13] and the present study), 609 faecal samples from wombats have been tested molecularly, and nine $(1.48 \%)$ were test-positive for Cryptosporidium and all were genetically very similar to $C$. fayeri (Fig. 2 and Additional file 2: Table S4). For the C. hominis, C. parvum and $C$. cuniculus clade, we found relatively few samples $(n=14)$ to be test-positive (by either SSU and/or gp60) for members of this clade compared with our previous study $(n=32)$ [13]. Considerably fewer samples were collected from rabbits $(n=97)$ than in the previous study $(n=263)$ [13]. The $C$. hominis positive (IbA10G2) from a sample from a wallaby from catchment MR was identical in gp60 sequence to a novel C. hominis genotype found in a human in Tasmania (GenBank accession no. KJ506839; [64]), and is the first report of C. hominis from a swamp wallaby. The genotypes of C. cuniculus characterised from kangaroos have been reported recently [33]. Within the $C$. canis clade, we identified Cryptosporidium consistent with $C$. canis in a faecal sample from an emu, which might relate to pseudo-parasitism, whereby the parasite is ingested and passed through the gastrointestinal tract of the host without establishing an infection. Typically, emus eat plants and insects [65], which could have been contaminated with oocyst-containing faeces from feral dogs or foxes within catchment CA. Within the $C$. suis clade, we identified, for the first time, a novel $C$. suislike genotype in a sample from a deer. Cryptosporidium 
suis has been considered to be specific to pigs, but has also been found in cattle, rodents, humans and chimpanzees [8]. Cryptosporidium suis-like protists that differ by only a few bases in SSU have been detected in cattle and rats [66, 67]. Within the C. ubiquitum clade, we report, for the first time, a novel C. ubiquitum-like genotype from wombats (Fig. 2). The samples were collected from catchments CA (July 2013) and OS (November 2014). Cryptosporidium ubiquitum is considered an emerging human pathogen that has been found in a wide range of wildlife, including canids, deer, primates and rodents [8]. Within the $C$. macropodum clade, we identified $C$. macropodum-like genotypes in six swamp wallabies from catchments CA, OS and SV. Three of the six genotypes were novel. Although there seems to be a differentiation within the C. macropodum clade between the wallabies and kangaroos in the catchments, this difference does not carry through to brush-tailed wallabies in New South Wales, where three such wallabies had a $C$. macropodum genotype and two shared $C$. macropodumlike genotypes [68]. More extensive sampling and testing of samples from wallabies and kangaroos throughout Victoria and New South Wales would be needed to clarify whether particular macropod host-affiliations exist. Within the $C$. ryanae clade, C. ryanae is usually found in cattle, but has also been recorded in other ruminants, such as water buffaloes and roe deer [8]. Although C. ryanae was not detected in this study, multiple closely related C. ryanae-like and Cryptosporidium sp. "deer" genotypes were identified in deer. The majority of the cervines in the catchments studied here are sambar deer, but it is not possible to confidently distinguish scats of sambar from those of fallow and red deer without using molecular tools. Using such tools, the specific identification of the cervine hosts would assist in assessing host affiliations and genetic diversity within the C. ryanae complex.

The potential role of some Cryptosporidium genotypes (e.g. C. suis-like in deer and C. ubiquitum-like in wombat - GenBank accession nos. KU531660 and KU531681, respectively) as zoonotic agents remains to be proven (cf. $[8,69,70])$. Further investigations of the presence and/or distribution of Cryptosporidium genotypes in rabbits, deer (e.g. C. hominis, detected here, for the first time, in wallaby) and other native and introduced wildlife in Australia, particularly in areas surrounding water catchments, are necessary, not only to determine the significance of various host groups as primary sources, potential reservoirs and amplifiers of Cryptosporidium for transmission to humans, but also to establish the mode(s) of transmission among reservoir animal hosts and how infection is maintained in wild animal populations $[8,62]$. The present results emphasize the need for increased investigation into the true host ranges of all Cryptosporidium species infecting wild and domesticated animals not yet studied.
Of the currently eight recognised species of Giardia, G. duodenalis is responsible for human disease [71-73], with isolates linked to sub-assemblages AI, AII, BIII and BIV, considered to be most commonly infective to humans, whereas sub-assemblages AIII, AIV, BI and BII are recognised to be infective to animals other than humans [72, 74]. Sub-assemblage AIII, in particular, is associated with deer and other wild ungulates $[72,75,76]$. All of the three genetic variants of tpi detected herein represented assemblage $\mathrm{A}$; two have been reported previously (e.g. GenBank accession nos. KU531708 and KU531707) (cf. [13]), and one is novel (accession no. KU531709). Since assemblages A and B of G. duodenalis appear to represent the greatest zoonotic risk, given their presence in humans, livestock and companion animals [71, 72, 74, 77], studying genetic variability within/among Giardia isolates is pivotal to inferring the zoonotic potential of distinct genotypes within this genus of parasite.

\section{Conclusions}

The present study has provided detailed insights into the taxa of Cryptosporidium and Giardia in animals in key water catchments in Victoria. The genetic analyses indicated that $1.92 \%$ of the 4,256 faecal samples contained Cryptosporidium or Giardia that matched species, genotypes or assemblages with the potential to infect humans. In addition, a number of new sequence records, which did not match any previously published genotypes, were identified. As nothing is known about the zoonotic potential of these new variants of Cryptosporidium and Giardia, future work should establish whether they are found in humans in Australia. Although the focus of the present study was on vast water catchment areas in south-eastern Australia, the research findings and the approach taken have considerable implications for other protected wilderness catchment areas around the world that supply unfiltered drinking water to millions of people.

\section{Additional files}

Additional file 1: Table S1. Number of faecal samples examined from each host/location combination. Table S2. Total number of each Cryptosporidium genotype sampled from each catchment. Table S3. Total number of each Cryptosporidium genotype sampled from each host. (DOCX $47 \mathrm{~kb}$ )

Additional file 2: Table S4. Pairwise comparisons of the SSU gene sequences among Cryptosporidium genotypes. Table S5. Pairwise comparisons of the gp60 gene sequences among Cryptosporidium genotypes. Table S6. Pairwise comparisons of the tpi gene sequences among Cryptosporidium genotypes. (XLSX 29 kb)

\section{Abbreviations}

AIC, Akaike information criteria; Bl, Bayesian inference; gp60, 60 kDa glycoprotein gene; MCMC, Monte Carlo Markov Chain; ORF, open reading frame; pp, posterior probability; SSCP, single-strand confirmation polymorphism; SSU, small subunit of ribosomal RNA; tpi, triose-phosphate isomerase gene 


\section{Acknowledgements}

Khrisdiana Putri provided technical assistance. Support from the Victoria Life Sciences Computation Initiative, Australia (VLSCl; grant no. VR0007) on its Peak Computing Facility at the University of Melbourne, Australia, an initiative of the Victorian Government, Australia is gratefully acknowledged (RBG).

\section{Funding}

Funding from the Australian Research Council (grant no. LP130100209) and Melbourne Water is gratefully acknowledged, as is support from the National Health and Medical Research Council (NHMRC) of Australia.

\section{Availability of data and material}

Nucleotide sequences reported in this paper are available in the GenBank database under accession nos. KU531647-KU531718.

\section{Authors' contributions}

Undertook the study and data analysis: AVK. Contributed to sample collection, analyses and/or interpretation: AVK, SH, ARJ and RBG. Wrote the paper: AVK and RBG with input from ARJ. Grant funding: RBG and ARJ. Supervision of project: RBG. All authors read and approved the final version of the manuscript.

\section{Competing interests}

The authors declare that they have no competing interests.

\section{Consent for publication}

Not applicable.

\section{Ethics approval and consent to participate}

Not applicable.

\section{Author details}

${ }^{1}$ Faculty of Veterinary and Agricultural Sciences, The University of Melbourne, Parkville, Victoria 3010, Australia. ${ }^{2}$ Melbourne Water, Docklands, Victoria 3001 Australia. ${ }^{3}$ The Walter and Eliza Hall Institute, Parkville, Victoria 3052, Australia.

Received: 5 April 2016 Accepted: 25 May 2016

Published online: 01 June 2016

\section{References}

1. WHO. Guidelines for drinking water quality: Cryptosporidium. New York: WHO Chron; 2006. p. 138.

2. Hunter PR, MacDonald AM, Carter RC. Water supply and health. PLoS Med. 2010;7(11):e1000361

3. Liu L, Johnson HL, Cousens S, Perin J, Scott S, Lawn JE, Rudan I, Campbell H, Cibulskis R, Li M, et al. Global, regional, and national causes of child mortality: an updated systematic analysis for 2010 with time trends since 2000. Lancet 2012;379(9832):2151-61

4. Kotloff KL, Nataro JP, Blackwelder WC, Nasrin D, Farag TH, Panchalingam S, Wu Y, Sow SO, Sur D, Breiman RF, et al. Burden and aetiology of diarrhoea disease in infants and young children in developing countries (the Global Enteric Multicenter Study, GEMS): a prospective, case-control study. Lancet. 2013;382(9888):209-22.

5. Striepen B. Parasitic infections: Time to tackle cryptosporidiosis. Nature 2013;503(7475):189-91

6. Lane S, Lloyd D. Current trends in research into the waterborne parasite Giardia. Crit Rev Microbiol. 2002;28(2):123-47.

7. Checkley W, White AC, Jr., Jaganath D, Arrowood MJ, Chalmers RM, Chen XM, Fayer R, Griffiths JK, Guerrant RL, Hedstrom L, et al. A review of the global burden, novel diagnostics, therapeutics, and vaccine targets for Cryptosporidium. Lancet Infect Dis. 2015;15(1):85-94.

8. Zahedi A, Paparini A, Jian F, Robertson I, Ryan U. Public health significance of zoonotic Cryptosporidium species in wildlife: critical insights into better drinking water management. Int J Parasitol Parasit Wildl. 2015;5:88-109.

9. Rendtorff RC. The experimental transmission of human intestinal protozoan parasites. II. Giardia lamblia cysts given in capsules. Am J Hyg. 1954;59(2): 209-20.

10. DuPont $\mathrm{HL}$, Chappell $\mathrm{CL}$, Sterling $\mathrm{CR}$, Okhuysen PC, Rose JB, Jakubowski W The infectivity of Cryptosporidium parvum in healthy volunteers. N Engl J Med. 1995;332(13):855-9.

11. Betancourt WQ, Rose JB. Drinking water treatment processes for removal of Cryptosporidium and Giardia. Vet Parasitol. 2004;126(1-2):219-34.
12. MacKenzie WR, Schell WL, Blair KA, Addiss DG, Peterson DE, Hoxie NJ, Kazmierczak JJ, Davis JP. Massive outbreak of waterborne Cryptosporidium infection in Milwaukee, Wisconsin: recurrence of illness and risk of secondary transmission. Clin Infect Dis. 1995:21(1):57-62.

13. Nolan MJ, Jex AR, Koehler AV, Haydon SR, Stevens MA, Gasser RB. Molecularbased investigation of Cryptosporidium and Giardia from animals in water catchments in southeastern Australia. Water Res. 2013;47(5):1726-40.

14. Chalmers RM, Ferguson C, Cacciò S, Gasser RB, Abs El-Osta YG, Heijnen L, Xiao L, Elwin K, Hadfield S, Sinclair M, et al. Direct comparison of selected methods for genetic categorisation of Cryptosporidium parvum and Cryptosporidium hominis species. Int J Parasitol. 2005;35(4):397-410.

15. Ferguson C, Deere D, Sinclair M, Chalmers RM, Elwin K, Hadfield S, Xiao L, Ryan UM, Gasser RB, Abs EL-Osta YG. Meeting report: application of genotyping methods to assess risks from Cryptosporidium in watersheds. Environ Health Perspect. 2006:114(3):430-4.

16. Gasser RB, Hu M, Chilton NB, Campbell BE, Jex AJ, Otranto D, Cafarchia C, Beveridge I, Zhu X. Single-strand conformation polymorphism (SSCP) for the analysis of genetic variation. Nat Protoc. 2006;1(6):3121-8.

17. Australian drinking water guidelines 6. 2011. http://www.nhmrc.gov.au/ guidelines-publications/eh52 Version 3.1. Access March 2015.

18. WHO. Guidelines for drinking-water quality. Fourth editionth ed. Geneva: World Health Organization; 2011. http://www.who.int/water_sanitation_ health/publications/2011/dwq_guidelines/en/.

19. Triggs B. Tracks, scats and other traces: a field guide to Australian mammals. South Melbourne: Oxford University Press; 2004

20. Dalén L, Götherström A, Angerbjörn A. Identifying species from pieces of faeces. Conserv Genet. 2004:5(1):109-11.

21. Sulaiman IM, Fayer R, Bern C, Gilman RH, Trout JM, Schantz PM, Das P, Lal $A A$, Xiao $L$ Triosephosphate isomerase gene characterization and potentia zoonotic transmission of Giardia duodenalis. Emerg Infect Dis. 2003:9(11): 1444-52.

22. Xiao L, Morgan UM, Limor J, Escalante A, Arrowood M, Shulaw W, Thompson R, Fayer R, Lal AA. Genetic diversity within Cryptosporidium parvum and related Cryptosporidium species. Appl Environ Microbiol. 1999;65(8):3386-91.

23. Nolan MJ, Jex AR, Pangasa A, Young ND, Campbell AJ, Stevens M, Gasser $\mathrm{RB}$. Analysis of nucleotide variation within the triose-phosphate isomerase gene of Giardia duodenalis from sheep and its zoonotic implications. Electrophoresis. 2010;31(2):287-98.

24. Ryan UM, Xiao L, Read C, Zhou L, Lal AA, Pavlasek I. Identification of nove Cryptosporidium genotypes from the Czech Republic. Appl Environ Microbiol. 2003;69(7):4302-7.

25. Strong WB, Gut J, Nelson RG. Cloning and sequence analysis of a highly polymorphic Cryptosporidium parvum gene encoding a 60-kilodalton glycoprotein and characterization of its 15-and 45-kilodalton zoite surface antigen products. Infect Immun. 2000;68(7):4117-34.

26. Mallon ME, MacLeod A, Wastling JM, Smith H, Tait A. Multilocus genotyping of Cryptosporidium parvum Type 2: population genetics and sub-structuring Infect Genet Evol. 2003;3(3):207-18

27. Alves M, Xiao L, Sulaiman I, Lal AA, Matos O, Antunes F. Subgenotype analysis of Cryptosporidium isolates from humans, cattle, and zoo ruminants in Portugal. J Clin Microbiol. 2003;41(6):2744-7.

28. Kearse M, Moir R, Wilson A, Stones-Havas S, Cheung M, Sturrock S, Buxton S, Cooper A, Markowitz S, Duran C, et al. Geneious Basic: an integrated and extendable desktop software platform for the organization and analysis of sequence data. Bioinformatics. 2012;28(12):1647-9.

29. Edgar RC. MUSCLE: multiple sequence alignment with high accuracy and high throughput. Nucleic Acids Res. 2004;32(5):1792-7.

30. Maddison W, Maddison D. Mesquite: A modular system for evolutionary analysis. v2.75. 2011

31. Huelsenbeck JP, Ronquist F. MRBAYES: Bayesian inference of phylogenetic trees. Bioinformatics. 2001;17(8):754-5.

32. Darriba D, Taboada GL, Doallo R, Posada D. jModelTest 2: more models, new heuristics and parallel computing. Nat Methods. 2012;9(8):772.

33. Koehler AV, Whipp MJ, Haydon SR, Gasser RB. Cryptosporidium cuniculus-new records in human and kangaroo in Australia. Parasit Vectors. 2014;7(492):1-3.

34. Jiang J, Alderisio KA, Xiao L. Distribution of Cryptosporidium genotypes in storm event water samples from three watersheds in New York. Appl Environ Microbiol. 2005:71(8):4446-54.

35. Feng Y, Alderisio KA, Yang W, Blancero LA, Kuhne WG, Nadareski CA, Reid M, Xiao L. Cryptosporidium genotypes in wildlife from a New York watershed. Appl Environ Microbiol. 2007;73(20):6475-83. 
36. Ziegler PE, Wade SE, Schaaf SL, Stern DA, Nadareski CA, Mohammed HO Prevalence of Cryptosporidium species in wildlife populations within a watershed landscape in southeastern New York State. Vet Parasitol. 2007;147(1-2):176-84.

37. Power ML, Slade MB, Sangster NC, Veal DA. Genetic characterisation of Cryptosporidium from a wild population of eastern grey kangaroos Macropus giganteus inhabiting a water catchment. Infect Genet Evol. 2004;4(1):59-67.

38. Power ML, Sangster NC, Slade MB, Veal DA. Patterns of Cryptosporidium oocyst shedding by eastern grey kangaroos inhabiting an Australian watershed. Appl Environ Microbiol. 2005;71(10):6159-64.

39. Cox P, Griffith M, Angles M, Deere D, Ferguson C. Concentrations of pathogens and indicators in animal feces in the Sydney watershed. Appl Environ Microbiol. 2005;71(10):5929-34.

40. Ryan UM, Read C, Hawkins P, Warnecke M, Swanson P, Griffith M, Deere D Cunningham M, Cox P. Genotypes of Cryptosporidium from Sydney water catchment areas. J Appl Microbiol. 2005;98(5):1221-9.

41. Black M, McAnulty J. The investigation of an outbreak of cryptosporidiosis in New South Wales in 2005. N S W Public Health Bull. 2006;17(5-6):76-9.

42. $\mathrm{Ng} J$, Yang R, Whiffin V, Cox P, Ryan UM. Identification of zoonotic Cryptosporidium and Giardia genotypes infecting animals in Sydney's water catchments. Exp Parasitol. 2011:128(2):138-44.

43. Buckley R, Warnken W. Giardia and Cryptosporidium in pristine protected catchments in central eastern Australia. AMBIO. 2003:32(2):84-6.

44. Cinque K, Stevens MA, Haydon SR, Jex AR, Gasser RB, Campbell BE. Investigating public health impacts of deer in a protected drinking water supply watershed. Water Sci Tech. 2008;58(1):127-32.

45. McCarthy S, Ng J, Gordon C, Miller R, Wyber A, Ryan UM. Prevalence of Cryptosporidium and Giardia species in animals in irrigation catchments in the southwest of Australia. Exp Parasitol. 2008;118(4):596-9.

46. van Dijk Al, Beck HE, Crosbie RS, Jeu RA, Liu YY, Podger GM, Timbal B, Viney NR. The Millennium Drought in southeast Australia (2001-2009): Natural and human causes and implications for water resources, ecosystems, economy, and society. Water Resour Res. 2013;49(2):1040-57.

47. Morgan U, Xiao L, Sulaiman I, Weber R, Lal AA, Thompson RC, Deplazes P. Which genotypes/species of Cryptosporidium are humans susceptible to? J Eukaryot Microbiol. 1999;46(5):42S-3.

48. Cacciò S. Molecular epidemiology of human cryptosporidiosis. Parassitologia. 2005:47(2):185-92.

49. Xiao L, Feng Y. Zoonotic cryptosporidiosis. FEMS Immunol Med Microbiol. 2008:52(3):309-23.

50. Cacciò SM, Putignani L. Epidemiology of human cryptosporidiosis. In: Cacciò SM, Widmer G, editors. Cryptosporidium: Parasite and Disease. Vienna: Springer; 2014. p. 43-79.

51. Ryan U, Fayer R, Xiao L. Cryptosporidium species in humans and animals: current understanding and research needs. Parasitology. 2014;141(13):1667-85.

52. Karanis $P$, Kourenti $C$, Smith $H$. Waterborne transmission of protozoan parasites: a worldwide review of outbreaks and lessons learnt. J Water Health. 2007;5(1): $1-38$.

53. Baldursson S, Karanis P. Waterborne transmission of protozoan parasites: Review of worldwide outbreaks-an update 2004-2010. Water Res. 2011; 45:6603-14

54. Chalmers RM, Robinson G, Elwin K, Hadfield SJ, Xiao L, Ryan UM, Modha D Mallaghan C. Cryptosporidium rabbit genotype, a newly identified human pathogen. Emerg Infect Dis. 2009;15(5):829-30.

55. Chalmers RM, Elwin K, Hadfield SJ, Robinson G. Sporadic human cryptosporidiosis caused by Cryptosporidium cuniculus, United Kingdom, 2007-2008. Emerg Infect Dis. 2011;17(3):536-8.

56. Elwin K, Hadfield SJ, Robinson G, Crouch ND, Chalmers RM. Cryptosporidium viatorum n. sp. (Apicomplexa: Cryptosporidiidae) among travellers returning to Great Britain from the Indian subcontinent, 2007-2011. Int J Parasitol. 2012; 42(7):675-82.

57. Fayer R. Taxonomy and species delimitation in Cryptosporidium. Exp Parasitol. 2010;124(1):90-7.

58. Li N, Xiao L, Alderisio K, Elwin K, Cebelinski E, Chalmers R, Santin M, Fayer R, Kvac M, Ryan UM, et al. Subtyping Cryptosporidium ubiquitum, a zoonotic pathogen emerging in humans. Emerg Infect Dis. 2014;20(2):217-24

59. Lucio-Forster A, Griffiths JK, Cama VA, Xiao L, Bowman DD. Minimal zoonotic risk of cryptosporidiosis from pet dogs and cats. Trends Parasitol. 2010;26(4):174-9.
60. Waldron LS, Cheung-Kwok-Sang C, Power ML. Wildlife-associated Cryptosporidium fayeri in human, Australia. Emerg Infect Dis. 2010;16(12): 2006-7.

61. Power M. Epidemiology of Cryptosporidium in eastern grey kangaroos Macropus giganteus inhabiting a Sydney water catchment, PhD Macquarie University. 2002.

62. Ryan UM, Power M. Cryptosporidium species in Australian wildlife and domestic animals. Parasitology. 2012;1(1):1-16.

63. Borchard P, Wright IA, Eldridge DJ. Wombats and domestic livestock as potential vectors of Cryptosporidium and Giardia in an agricultural riparian area. Aust J Zool. 2010;58(3):150-3.

64. Koehler AV, Whipp M, Hogg G, Haydon SR, Stevens MA, Jex AR, Gasser RB. First genetic analysis of Cryptosporidium from humans from Tasmania, and identification of a new genotype from a traveller to Bali. Electrophoresis. 2014;35(18):2600-7.

65. Quin B. Diet and habitat of emus Dromaius novaehollandiae in the Grampians Ranges, south-western Victoria. Emu. 1996;96(2):114-22.

66. Ng-Hublin JS, Combs B, MacKenzie B, Ryan U. Human cryptosporidiosis diagnosed in Western Australia: a mixed infection with Cryptosporidium meleagridis, the Cryptosporidium Mink Genotype, and an unknown Cryptosporidium species. J Clin Microbiol. 2013;51(7):2463-5.

67. Ma J, Li P, Zhao X, Xu H, Wu W, Wang Y, Guo Y, Wang L, Feng Y, Xiao L. Occurrence and molecular characterization of Cryptosporidium spp. and Enterocytozoon bieneusi in dairy cattle, beef cattle and water buffaloes in China. Vet Parasitol. 2015;207(3-4):220-7.

68. Vermeulen ET, Ashworth DL, Eldridge MD, Power ML. Diversity of Cryptosporidium in brush-tailed rock-wallabies (Petrogale penicillata) managed within a species recovery programme. Int J Parasitol Parasites Wildl. 2015;4(2):190-6.

69. Robinson G, Elwin K, Chalmers RM. Unusual Cryptosporidium genotypes in human cases of diarrhea. Emerg Infect Dis. 2008;14(11):1800-2.

70. Chalmers RM, Davies AP. Minireview: clinical cryptosporidiosis. Exp Parasitol. 2010;124(1):138-46.

71. Feng Y, Xiao L. Zoonotic potential and molecular epidemiology of Giardia species and giardiasis. Clin Microbiol Rev. 2011;24(1):110-40.

72. Ryan UM, Caccio SM. Zoonotic potential of Giardia. Int J Parasitol. 2013;43: 943-56.

73. Thompson RC, Ash A. Molecular epidemiology of Giardia and Cryptosporidium infections. Infect Genet Evol. 2015.

74. Monis PT, Andrews RH, Mayrhofer G, Ey PL. Genetic diversity within the morphological species Giardia intestinalis and its relationship to host origin. Infect Genet Evol. 2003;3(1):29-38.

75. Robertson LJ, Forberg T, Hermansen L, Hamnes IS, Gjerde B. Giardia duodenalis cysts isolated from wild moose and reindeer in Norway: genetic characterization by PCR-rflp and sequence analysis at two genes. J Wildl Dis. 2007:43(4):576-85.

76. Cacciò SM, Beck R, Lalle M, Marinculic A, Pozio E. Multilocus genotyping of Giardia duodenalis reveals striking differences between assemblages A and B. Int J Parasitol. 2008;38(13):1523-31.

77. Xiao L, Fayer R. Molecular characterisation of species and genotypes of Cryptosporidium and Giardia and assessment of zoonotic transmission. Int J Parasitol. 2008:38(11):1239-55.

\section{Submit your next manuscript to BioMed Central and we will help you at every step:}

- We accept pre-submission inquiries

- Our selector tool helps you to find the most relevant journal

- We provide round the clock customer support

- Convenient online submission

- Thorough peer review

- Inclusion in PubMed and all major indexing services

- Maximum visibility for your research

Submit your manuscript at www.biomedcentral.com/submit 